\title{
ANSIEDAD Y EXPRESIÓN GRÁFICA EN PACIENTES PEDIÁTRICOS HOSPITALIZADOS
}

\author{
Irene Acosta Pérez \\ Departamento de Psicología. Facultad de Enfermería de Cuenca, UCLM. irene.acostaperez@ucIm.es
}

Recepción Artículo: 27 octubre 2021 Admisión Evaluación: 27 octubre 2021 Informe Evaluador 1: 28 octubre 2021 Informe Evaluador 2: 29 octubre 2021 Aprobación Publicación: 30 octubre 2021

\section{RESUMEN}

Aun con el progreso llevado a cabo en la atención y políticas sanitarias, la hospitalización infantil sigue siendo un acontecimiento vital capaz de generar diversas alteraciones emocionales y físicas, siendo una de ellas la ansiedad. Existe un riesgo potencial de que se originen una serie de alteraciones emocionales y conductuales en el niño que modifiquen su desarrollo personal. Junto a los efectos emocionales, otro de los problemas de los niños hospitalizados es el de la comunicación, ya que les resulta difícil expresarse. La expresión gráfica del niño se halla en íntima relación con todo su desarrollo evolutivo y en especial con el de su pensamiento, con sus modos de expresión y comunicación. En este sentido, el dibujo puede ser una herramienta útil para que los niños hospitalizados relaten sus experiencias y sentimientos sin palabras. El objetivo de la presente investigación es conocer la ansiedad que genera la hospitalización en niños de 6 a 12 años de edad ingresados en 3 hospitales públicos de Caracas, Venezuela, y su relación con la expresión gráfica en dicha situación hospitalaria. El estudio fue cuasi-experimental sin grupo control, transversal, cuantitativo de tipo descriptivo, correlacional y comparativo. Los instrumentos utilizados fueron el Inventario de autoevaluación Ansiedad Estado-Rasgo en niños (STAIC) y el dibujo libre, tomando en cuenta los indicadores de ansiedad en el dibujo libre recopilados por Acosta y Crespo (2002). Los resultados mostraron que la experiencia de hospitalización es percibida como un evento vital estresante que genera ansiedad, así como correlaciones directas y significativas entre la ansiedad estado y la presencia de indicadores de ansiedad en el dibujo. Se concluye que el empleo del dibujo constituye una estrategia eficaz para explorar las percepciones de ansiedad que tienen los niños sobre la hospitalización, cuyo impacto requiere que se incluya la esfera emocional dentro del cuidado hospitalario. Se hace necesario detectar la ansiedad y desarrollar intervenciones para prevenir o disminuir sus efectos en el niño hospitalizado.

Palabras clave: paciente pediátrico; hospitalización; ansiedad; expresión gráfica; dibujo 


\section{ANSIEDAD Y EXPRESIÓN GRÁFICA EN PACIENTES PEDIÁTRICOS HOSPITALIZADOS}

\section{ABSTRACT}

Anxiety and graphic expression in hospitalized pediatric patients. Even with the progress made in health care and policies, child hospitalization continues to be a vital event capable of generating various emotional and physical alterations, one of them being anxiety. There is a potential risk that a series of emotional and behavioral alterations will originate in the child that modify their personal development. Along with the emotional effects, another problem of hospitalized children is that of communication, since it is difficult for them to express themselves. The graphic expression of the child is in intimate relationship with all its evolutionary development and especially with that of its thought, with its modes of expression and communication. In this sense, drawing can be a useful tool for hospitalized children to relate their experiences and feelings without words. The objective of this research is to know the anxiety generated by hospitalization in children between 6 and 12 years of age admitted to 3 public hospitals in Caracas, Venezuela, and its relationship with their graphic expression in that situation. A quasi-experimental study without a control group, cross-sectional, quantitative, descriptive, correlational and comparative has been designed. The instruments used were the State-Trait Anxiety SelfAssessment Inventory in Children (STAIC), and free drawing, taking into account the anxiety indicators in free drawing collected by Acosta and Crespo (2002). The results showed that the hospitalization experience is perceived as a stressful life event that generates anxiety, as well as direct and significant correlations between state anxiety and the presence of anxiety indicators in the drawing. It is concluded that the use of drawing constitutes an effective strategy to explore children's perceptions of anxiety about hospitalization, the impact of which requires that the emotional sphere be included in hospital care. It is necessary to detect anxiety and develop interventions to prevent or reduce its effects on hospitalized children.

Keywords: pediatric patient; hospitalization; anxiety; graphic expresión; drawing

\section{INTRODUCCIÓN}

Según Aguilera y Whetsell (2007) la percepción de los niños sobre su propia experiencia de hospitalización puede exceder sus capacidades para poder resolverla y afrontarla, Ilevándolos a presentar ansiedad. Junto a estos efectos emocionales, los niños en situación hospitalaria presentan dificultades para comunicarse, expresarse y relacionarse (Serradas, 2006) en mayor medida que las que puedan tener en su casa 0 en la escuela; lo cual puede generar miedos, ansiedad y disminución de la autoconfianza y de la autoestima, y dificultar la aceptación del tratamiento y por tanto la mejoría con respecto a la causa del ingreso hospitalario (Soares y Correa, 2011).

La enfermedad y la hospitalización infantil son eventos estresantes que originan cambios en la vida y rutinas del niño, teniendo que afrontar los sucesos relacionados a su tratamiento en el hospital. En este sentido, Aguilar Morales (2011) indica que el contacto con los servicios de salud genera ansiedad en los niños.

Spielberger y Díaz (1975) señalan que la ansiedad es una reacción emocional adaptativa y desagradable producida por un estímulo externo, que es considerado por el individuo como amenazante, produciendo cambios fisiológicos y conductuales. Las sensaciones subjetivas de aprensión, tensión y preocupación son percibidas por la persona como transitorias, y fluctúan en el tiempo (Fernández y López, 2011).

Según Spielberg y Díaz (1975) la ansiedad se presenta de dos formas. La ansiedad-rasgo, definida como la respuesta característica del niño ante el estímulo ansioso; sería por ejemplo la tendencia a responder a la situación de hospitalización con aumento de la ansiedad. Por otra parte, la ansiedad-estado sería el estado emocional transitorio del niño como respuesta ante la hospitalización, caracterizada por elementos de tensión, debido al aumento de la actividad del sistema nervioso autónomo (Nishiyama, Matsukawa y Hanaoka, 1998).

La investigación en este área en los últimos años se ha orientado hacia la búsqueda de soluciones y estrategias de afrontamiento de la situación de hospitalización en los pacientes pediátricos, siendo una de ellas la interpretación del dibujo libre; ya que cuando hay dificultades para que el niño se exprese, el dibujo puede servir de lenguaje común para comunicarse. En los niños, la expresión gráfica está profundamente relacionada con todo su desarrollo evolutivo, pero especialmente con el desarrollo del pensamiento, los modos de expresión y la comunicación, tal y como señalan Escobar y Romero (2003). En los adultos predomina la expresión verbal, pero 
Ios niños utilizan otros recursos para expresar su mundo emocional mediante medios y lenguajes distintos; siendo el dibujo uno de ellos, en los que el niño hospitalizado puede representar sin palabras los cambios afectivos, emocionales, psicológicos y físicos que experimenta (López y Ruiz, 2016; Silva, 2012). Arguelles, García y Agramonte (2006) catalogan el dibujo como un medio de comunicación fundamental en estas edades. .A través del dibujo, se puede valorar la ansiedad relacionada con la hospitalización y además puede ser útil para hablar de asuntos más complejos con el paciente pediátrico, pudiendo llegar a convertirse en el termómetro del estado de ánimo del niño (Ávila Espada, 1997).

Zafra et al. (2018) señalan que a través de los dibujos, se puede interpretar la influencia de la enfermedad y la hospitalización sobre los pacientes ingresados, lo que podría contribuir a un replanteamiento del enfoque de su atención tanto física como psicoemocional.

\section{OBJETIVOS DE LA INVESTIGACIÓN}

El objetivo general de la presente investigación es conocer la ansiedad estado - rasgo y su relación con la expresión gráfica en pacientes pediátricos hospitalizados. Los objetivos específicos son:

Evaluar la Ansiedad - Estado en pacientes pediátricos hospitalizados,

Evaluar la Ansiedad - Rasgo en pacientes pediátricos hospitalizados.

Evaluar los indicadores de ansiedad en el dibujo libre en pacientes pediátricos hospitalizados.

Establecer las posibles relaciones entre la Ansiedad Estado - Rasgo y los indicadores de ansiedad presentes en el dibujo libre en pacientes pediátricos hospitalizados.

\section{MUESTRA Y/O PARTICIPANTES}

Para desarrollar este estudio se seleccionaron al azar pacientes hospitalizados en los Servicios de Pediatría de los siguientes hospitales en Venezuela: Hospital ortopédico infantil (Distrito Capital), Hospital J.M. de los Ríos (Distrito Capital) y Hospital Victorino Santaella Ruiz (Estado Miranda).

La muestra estuvo constituida por 26 niños hospitalizados. Los criterios de selección fueron que el paciente tuviera 3 o más días hospitalizado, y una edad comprendida entre los 6 y 12 años, ya que dicho rango abarca la escolarización primaria cuyo final coincide, además, con la edad en que los pacientes suelen dejar de ser hospitalizados en el servicio de pediatría. En esta etapa los niños hacen avances notables en su habilidad para comprender su mundo y para pensar de manera lógica. Piaget (1950; en Craig, 1997) postulaba que durante este período se pasa a la etapa del desarrollo cognoscitivo de las acciones concretas; por esto, los niños desarrollan su capacidad para hacer inferencias lógicas, reflexionar sobre las transformaciones físicas, realizar operaciones mentales reversibles y, en general, formular hipótesis sobre el mundo material.

La media de edad de los participantes fue de 9.12 años, con mayor porcentaje masculino (61.53\%) y un promedio de hospitalización de 3.5 días.

\section{METODOLOGÍA Y/O INSTRUMENTOS UTILIZADOS}

El estudio fue cuasi-experimental sin grupo control, transversal, cuantitativo de tipo descriptivo, correlacional y comparativo. Para la recolección de los datos se obtuvieron los permisos necesarios para llevar a cabo la recogida de datos en cada hospital, así como el consentimiento informado de los padres de cada participante. El análisis de los datos se realizó mediante el paquete estadístico SPSS versión 11.0.1.

Se inició con un proceso de observación participante encaminado a un proceso de acercamiento con los niños y sus familiares, con el fin de establecer una adecuada comunicación antes de la recopilación de los datos. Los instrumentos fueron aplicados durante el segundo trimestre del 2002, de forma individual en las habitaciones de los niños. Para evaluar el nivel de ansiedad se utilizó el Cuestionario de Ansiedad Estado-Rasgo en niños (STAIC), creado por Spielberger et al. (1973) y adaptado para la población española por Seisdedos (1990). Está compuesto por dos escalas independientes, cada una conformada por 20 ítems con una escala de respuesta tipo 
Likert de: Nada (1), Algo (2), Bastante (3) y Mucho (4). La primera evalúa la Ansiedad Estado (AE), o el nivel de ansiedad del niño en un momento determinado (estado transitorio: aquellos sentimientos de aprensión, tensión,y preocupación que fluctúan y varían en intensidad con el tiempo ), y la segunda identifica la Ansiedad Rasgo (AR), o cómo se siente el niño en general (rasgo latente: diferencias relativamente estables de propensión a la ansiedad, es decir, diferencias entre los niños en su tendencia a mostrar estados de ansiedad).

El instrumento puede ser usado en población normal o clínica. Si bien está diseñado para niños entre 9 y 15 años de edad preferentemente, es aplicable a niños mayores de 5 años (Ahmed, Farrell, Parris Karla; 2011).

Para la corrección del STAIC se obtiene una puntuación de ansiedad estado y una puntuación de ansiedad rasgo, sumando cada uno de los ítems de cada subescala. La puntuación puede oscilar entre 20 (poca ansiedad) y 60 (mucha ansiedad) en ambas escalas, y las puntuaciones directas se transforman en puntuaciones normalizadas. La fiabilidad de la prueba mediante el procedimiento de las dos mitades (pares-impares) muestra un índice de 0,88 y 0,90 en AE; y, 0,83 y 0,86 en AR para hombres y mujeres respectivamente.

La evaluación de los indicadores de ansiedad en el dibujo libre se llevó a cabo solicitando a cada participante que realizara el dibujo que quisiera y tomando en cuenta sus actitudes, comentarios y relatos durante la realización del mismo. Posteriormente los dibujos fueron analizados siguiendo el listado de Indicadores de ansiedad en el dibujo libre recopilado por Acosta y Crespo (2002), los cuales se recogen en la Tabla 1.

Tabla 1.

Indicadores de ansiedad evaluados en el dibujo libre

\begin{tabular}{|l|l|l|}
\hline \multicolumn{1}{|c|}{ Generales } & \multicolumn{1}{|c|}{ Figura humana } & \multicolumn{1}{c|}{ Árbol } \\
\hline $\begin{array}{l}\text { Dibujar una parte pequeña, } \\
\text { borrar y luego hacerla muy } \\
\text { grande }\end{array}$ & Boca cerrada & $\begin{array}{l}\text { Tronco dibujado con } \\
\text { líneas débiles, esbozadas, } \\
\text { punteadas que no } \\
\text { aparecen en ninguna otra } \\
\text { parte }\end{array}$ \\
\hline Dibujos pequeños & $\begin{array}{l}\text { Extremidades rígidas y } \\
\text { orientadas forzadamente } \\
\text { hacia el eje del cuerpo }\end{array}$ & $\begin{array}{l}\text { Línea del suelo } \\
\text { fuertemente señalada }\end{array}$ \\
\hline $\begin{array}{l}\text { Líneas livianas } \\
\text { Líneas pesadas }\end{array}$ & $\begin{array}{l}\text { Contorno de dimensiones } \\
\text { desmesuradas }\end{array}$ & $\begin{array}{l}\text { Mezcla inarmónica de } \\
\text { colores cálidos }\end{array}$ \\
\hline $\begin{array}{l}\text { Líneas quebradas, cortas, } \\
\text { indecisas, esbozadas, o que } \\
\text { tienen continuidad sólo } \\
\text { varias veces }\end{array}$ & $\begin{array}{l}\text { Interrupción del dibujo en } \\
\text { determinado punto }\end{array}$ & $\begin{array}{l}\text { Reforzamiento de un color } \\
\text { volviendo sobre él varias } \\
\text { veces }\end{array}$ \\
\hline $\begin{array}{l}\text { Borrones } \\
\text { Omisión de boca }\end{array}$ & Omisión de cuerpo & \\
\hline
\end{tabular}




\begin{tabular}{|l|l|l|}
\hline Sombreado & Omisión de brazos & \\
\hline $\begin{array}{l}\text { Preocupación por la } \\
\text { simetría }\end{array}$ & Omisión de piernas & \\
\hline $\begin{array}{l}\text { Figuras que se unen al } \\
\text { borde del papel }\end{array}$ & Omisión de ojos & \\
\hline $\begin{array}{l}\text { Repetición de } \\
\text { determinados objetos }\end{array}$ & Piernas juntas & \\
\hline Nubes, lluvia, nieve & Comisura de la boca caída & \\
\hline Aves & & \\
\hline
\end{tabular}

\section{RESULTADOS Y DISCUSIÓN}

Ansiedad Estado: el 73\% de los niños presentó AE. Los ítems con mayores porcentajes de respuestas en la escala de AE fueron: Estoy desosegado, intranquilo (62\%), Me siento alterado (57,2\%), me siento muy atado (como atrapado) (53,7\%), Me siento aturdido y sobreexcitado (46,4\%), y Estoy preocupado por posibles desgracias futuras (42,8\%). Estos ítems son la expresión de la inadecuación en el ambiente hospitalario, de acuerdo a Nishiyama et al. (1998).

Ansiedad Rasgo: el 41\% de los niños hospitalizados presentó AR. Los ítems con mayores porcentajes de respuesta en la escala de AR fueron: Me siento triste (62.4\%), Me rodean y me molestan pensamientos sin importancia (53,3\%), Me preocupo demasiado por cosas sin importancia (51,5\%), y Siento ganas de llorar (43,6\%), en la línea de lo hallado por Alejos - Cerdán, A ráoz-Chavez y Castañeda - Chang (2003).

Dibujo libre: Se apreció buena aceptación de la técnica del dibujo, lo cual coincide con lo descrito por autores como Arguelles, García y Agramonte (2006) sobre la importancia del dibujo como canal de expresión emocional a estas edades. Todos los niños presentaron al menos un indicador de ansiedad en el dibujo libre. Los indicadores con mayor porcentaje de aparición fueron: líneas quebradas, cortas, indecisas, esbozadas, o que tienen continuidad sólo porque fueron reforzadas varias veces $(65 \%$,), dibujos pequeños $(53,2 \%)$, figuras que se unen al borde del papel (49,7\%), interrupción del dibujo en determinado punto (41,3\%), Reforzamiento de un color volviendo sobre él varias veces (37,5\%), dibujar una parte pequeña, borrar, y luego hacerla muy grande $(35,4 \%)$, líneas pesadas $(33,4 \%)$, borrones $(28,8 \%)$, nubes, Iluvia, nieve $(25,3 \%)$, preocupación por la simetría $(23 \%)$, líneas livianas (21,7\%), Línea del suelo fuertemente señalada (18,8\%), omisiones de boca, cuerpo, brazos, piernas u ojos (13\%). Esta importante presencia de indicadores de ansiedad en los dibujos de los pacientes, concuerda con lo señalado por López y Ruiz (2016) y Silva (2012) acerca de la utilidad e importancia del dibujo como recurso comunicativo en el niño para la expresión de sus emociones y percepciones; en cuanto a los diversos cambios a los que se enfrenta en la situación de hospitalización.

Relación entre Ansiedad Estado - Rasgo y Dibujo libre: no se encontraron correlaciones estadísticamente significativas entre la Ansiedad - Rasgo y los indicadores de ansiedad en el dibujo libre. Se hallaron correlaciones positivas y estadísticamente significativas entre la Ansiedad - Estado y 6 indicadores de ansiedad

en el dibujo libre, tal como se muestra en la tabla 2. Estos resultados pudieran ir en la línea de la idea de que la ansiedad en niños hospitalizados puede aparecer a partir de la percepción del desbordamiento de sus recursos (Aguilera y Whetsell, 2007), a partir del contacto con los servicios de salud que implica la propia hospitalización (Aguilar Morales, 2011). 


\section{ANSIEDAD Y EXPRESIÓN GRÁFICA EN PACIENTES PEDIÁTRICOS HOSPITALIZADOS}

Tabla 2.

Correlaciones significativas entre Ansiedad - Estado y los indicadores de ansiedad en el dibujo libre

\begin{tabular}{|l|c|}
\hline Indicadores de Ansiedad en el dibujo libre & Correlación con A-E \\
\hline Dibujos pequeños (indicador general) & $0.46^{*}$ \\
\hline $\begin{array}{l}\text { Líneas quebradas, cortas, indecisas, } \\
\text { esbozadas, o que tienen continuidad sólo } \\
\text { porque fueron reforzadas varias veces } \\
\text { (indicador general) }\end{array}$ & $0.52^{* *}$ \\
\hline $\begin{array}{l}\text { Figuras que se unen al borde del papel } \\
\text { (indicador general) }\end{array}$ & $0.24^{* *}$ \\
\hline $\begin{array}{l}\text { Interrupción del dibujo en determinado } \\
\text { punto (figura humana) }\end{array}$ & $0.39^{*}$ \\
\hline $\begin{array}{l}\text { Reforzamiento de un color volviendo sobre } \\
\text { él varias veces (árbol) }\end{array}$ & $0.16^{*}$ \\
\hline
\end{tabular}

Nota. * La correlación es significativa al nivel 0.05 (bilateral). ** La correlación es significativa al nivel 0.01 (bilateral).

\section{CONCLUSIONES}

La ansiedad es difícil de cuantificar dado su carácter variable y subjetivo. Tomando esto en cuenta, en el presente estudio se ha podido constatar que la hospitalización constituye una situación vital estresante ante la que Ios niños configuran percepciones y emociones negativas; viviéndola como una situación amenazante.

La técnica del dibujo ha sido eficaz para explorar las vivencias internas y la expresión emocional de los pacientes pediátricos sobre su situación hospitalaria, cuyo impacto requiere que se incluya la esfera emocional dentro del cuidado hospitalario. En cuanto a la relación

entre la ansiedad estado - rasgo y los indicadores de ansiedad en el dibujo libre, parece que este último podría ser útil a la hora de determinar la existencia de ansiedad de los niños ante la situación hospitalaria (ansiedad - estado); pero no de sus propias características personales relacionadas con la vivencia de la ansiedad (ansiedad - rasgo).

Se recomienda estudiar en mayor profundidad la utilidad del dibujo en el entorno hospitalario, así como sus características en los distintos momentos del proceso de hospitalización. La interpretación de los dibujos no cuenta aún con suficiente validación experimental, pero puede aportar información valiosa sobre el mundo psicoemocional de los niños y facilitar la comunicación con ellos en situaciones tan demandantes como el tener que permanecer ingresado en un hospital. Se hace necesario detectar la ansiedad y desarrollar intervenciones para prevenir o disminuir sus efectos en el niño hospitalizado.

\section{REFERENCIAS BIBLIOGRÁFICAS}

Acosta, I. y Crespo, S. (2002). Manifestaciones de ansiedad en pacientes pediátricos hospitalizados. (Trabajo de

fin de grado no publicado). Universidad Central de Venezuela.

Aguilar Morales, J.E. (2011). Preparación psicológica de niños para una intervención quirúrgica. Recuperado en

septiembre de 2021 de http://www.conductitlan.net/cirugia.htm

Ahmed, M.I., Farrell, M.A., Parrish K. y Karla A. (2011). Preoperative anxiety in children risk factors and 
nonpharmacological management. Middle East Journal of Anesthesiology 21(2), 153-164.

Alejos-Cerdán, M., Aráoz-Chávez, L.M., Castañeda- Chang, A.M. (2003).

Ansiedad-estado-rasgo en niños y adolescentes durante la hospitalización.

Psicocentro. http://www. psicocentro.com/cgi-bin/articulo_s.asp?texto=art3b002 Arguelles, T., García, R. y Agramonte, A. (2006). El dibujo como exponente de las

percepciones sobre la enfermedad en niños y niñas con diabetes mellitus. Revista Cubana de Endocrinología, 17(1).

Ávila Espada, A. (1997). Evaluación en psicología clínica: Estrategias cualitativas. Amarú Ediciones.

Craig, G. (1997). Desarrollo psicológico. Prentice - Hall Hispanoamericana.

Escobar, M.J. y Romero, K. (2003). Desarrollo de la representación del cuerpo humano y la familia en el niño preescolar a través de las artes: reflexiones teóricas. Educere, 6(21), 33-39.

Fernández A. y López I. (2011). Estrés en padres e hijos en la hospitalización infantil. Revista de psicología. Disponible en http://www.revistapediatria.cl/vol6num1/ 4.html

López, y Ruiz, (2016). Estrategias de adaptación a la hospitalización en niños preescolares.

(Trabajo de fin de grado no publicado). Universidad Autónoma de Madrid.

Nishiyama, T., Matsukawa, T. y Hanaoka K. (1998). The effects of age and gender on the optimal premedication dose of intramuscular midazolam. Anesthesia \& Analgesia, 86, 1103-1108.

Seisdedos, N. (1990). STAIC, Cuestionario de Autoevaluación. TEA Ediciones.

Silva Panez, G. (2012). Las vivencias de los niños hospitalizados desde sus dibujos y testimonios: un estudio cualitativo con enfoque de resiliencia y los derechos del niño hospitalizado. Instituto de Estudios Peruanos (IEP) : Fundación Telefónica.

Soares Albano, M. A. y Correa, I. (2011). Lectura de cuentos infantiles como estrategia de humanización en el cuidado del niño encamado en ambiente hospitalario Investigación y Educación en Enfermería, 29(3), 370380.

Spielberger, C. D. (1973). Inventario de Ansiedad Estado - Rasgo para niños, STAIC.

Consulting Psychologists Press.

Spielberger, C. y Díaz, R. (1975). Inventario de ansiedad, rasgo-estado IDARE. Librería Interacadémica.

Zafra Anta, M.A., Caballero Jiménez, E.M., Ruiz García, M., Medino Muñoz, J., Hindo Jazrawi, D. y Elipe Maldonado, C. (2018). Los sueños durante la hospitalización, valor de su narrativa plástica como forma de humanización de la asistencia. Revista Pediátrica de Atención Primaria, 20, 145-50. 
